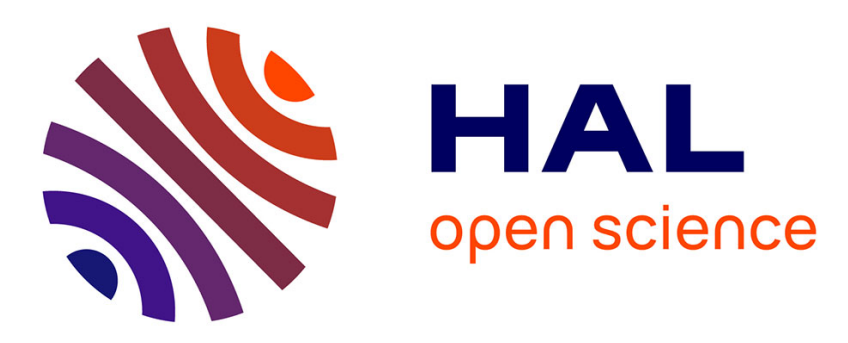

\title{
Modèles et mesures de l'influence de la communication : Nouvelles perspectives ouvertes par la psychologie sociale
}

Didier Courbet, Marie-Pierre Fourquet-Courbet

\section{- To cite this version:}

Didier Courbet, Marie-Pierre Fourquet-Courbet. Modèles et mesures de l'influence de la communication: Nouvelles perspectives ouvertes par la psychologie sociale. Revue internationale de psychosociologie et de gestion des comportements organisationnels, 2008, XI (25). sic_00001647

\section{HAL Id: sic_00001647 \\ https://archivesic.ccsd.cnrs.fr/sic_00001647}

Submitted on 10 Jan 2006

HAL is a multi-disciplinary open access archive for the deposit and dissemination of scientific research documents, whether they are published or not. The documents may come from teaching and research institutions in France or abroad, or from public or private research centers.
L'archive ouverte pluridisciplinaire HAL, est destinée au dépôt et à la diffusion de documents scientifiques de niveau recherche, publiés ou non, émanant des établissements d'enseignement et de recherche français ou étrangers, des laboratoires publics ou privés. 


\title{
Modèles et mesures de l'influence de la communication :
}

\section{Nouvelles perspectives ouvertes par la psychologie sociale}

\author{
Didier Courbet \\ Maître de Conférences HDR en sciences de l'information de la communication \\ Université de Nice-Sophia Antipolis, \\ Laboratoire de psychologie LPEQ et Equipe I3M (Information, Médias, Milieu, Médiation)
}

\author{
Marie-Pierre Fourquet-Courbet \\ Maître de Conférences en sciences de l'information de la communication \\ Université d'Avignon - Laboratoire Culture et Communication
}

\section{Résumé.}

La question de l'efficacité des actions de communication est au coeur des préoccupations des praticiens de la communication et du marketing. Pour concevoir des modèles d'aide à la décision, les chercheurs mobilisent souvent la psychologie sociale. L'objectif de l'article est de montrer comment de récentes recherches dans les domaines de la socio-cognition implicite et de la psychologie cognitive et linguistique ont été appliquées aux communications publicitaire et politique afin de mieux connaître, premièrement, le lien attitude-comportement, deuxièmement, les effets de la publicité vue rapidement et aussitôt oubliée, et, troisièmement, les traitements psychologiques en temps réel des récepteurs face à des messages.

Mots-clés : socio-cognition implicite, persuasion, attitude implicite, comportement du consommateur, internet, analyse de contenu.

\section{Titre et résumé en anglais}

Models and measurements of the persuasive communication influence:

New perspectives opened by social psychology

Summary. The question of the persuasive communication effectiveness is essential for the marketers. To design models of decision-making aid, the researchers often mobilize social psychology. The objective of the article is to show how recent research in the fields of implicit social cognition and cognitive and linguistic psychology was applied to advertising and political communication in order to better know, firstly, the attitude-behavior links; secondly, the effects of quickly seen messages and at once forgotten, and, thirdly, the "real time psychological treatments" of the receivers face messages. Titre et résumé en anglais provisoire

Key words: implicit social cognition, persuasion, implicit attitude, consumer behavior, Internet, content analysis. 


\title{
Modèles et mesures de l'influence de la communication : Nouvelles perspectives ouvertes par la psychologie sociale
}

\author{
Didier Courbet \\ Maître de Conférences HDR en sciences de l'information de la communication \\ Université de Nice-Sophia Antipolis, \\ Laboratoire de psychologie LPEQ et Equipe I3M (Information, Médias, Milieu, Médiation)
}

\author{
Marie-Pierre Fourquet-Courbet \\ Maître de Conférences en sciences de l'information de la communication \\ Université d'Avignon \\ Laboratoire Culture et Communication
}

Les questions relatives à l'efficacité et à l'impact des actions de communication sont au coeur des préoccupations des praticiens de la communication et du marketing. Ces questions fondamentales concernent aussi bien la communication commerciale que d'autres domaines de la communication comme la communication politique. La recherche académique a élaboré de multiples modèles et méthodes pour expliquer les effets et les processus d'influences psychologiques des individus ayant été en contact avec des messages commerciaux ou des discours politiques. Les praticiens disposent désormais de nombreux modèles théoriques d'aide à la décision et de méthodes leur permettant d'obtenir des informations pour optimiser la création des messages, l'élaboration des plans médias, les discours persuasifs... Même si les modèles sont souvent pluridisciplinaires, une grande partie des concepts et méthodes sont issus de la psychologie sociale.

L'objectif de cet article est de montrer comment de récentes recherches dans les domaines de la socio-cognition implicite et de la psychologie cognitive et linguistique peuvent être appliquées à un contexte de communication persuasive afin de proposer des solutions à certains problèmes auxquels se heurtent les praticiens et chercheurs travaillant dans la communication commerciale et la communication politique.

Quelques problèmes auxquels se heurtent les praticiens et les chercheurs en communication persuasive

\section{Deux problèmes théoriques}

Le premier problème théorique, récurrent dans la recherche et les pratiques de communication persuasive, concerne le lien entre les jugements, l'attitude et le comportement. En l'appliquant à un 
contexte de communication commerciale, nous présentons un récent modèle intégrateur, proposé par des psychologues sociaux cognitifs (modèle MODE, Fazio et Towles-Schwen, 1999 ; Schuette et Fazio, 1995). Dans la première partie de l'article, nous montrons comment ce modèle, jusqu'à présent ignoré dans la littérature marketing, contribue à mieux expliquer les processus reliant attitude à l'égard de la marque et comportement d'achat. Le deuxième problème théorique concerne la coexistence, chez un même individu, de deux types d'attitudes, parfois ouvertement opposées, à l'égard d'un même objet. Les praticiens le constatent fréquemment : lorsqu'une personne émet rapidement une attitude à l'égard d'une marque ou d'un produit, celle-ci est parfois différente de l'attitude émise de manière plus réfléchie. Depuis quelques années, les chercheurs en sociocognition implicite ont étudié cette double attitude et montré le rôle et l'importance des automatismes dans les jugements. Dans la deuxième partie, à l'aide d'une recherche que nous avons menée dans le domaine de la communication persuasive (Courbet et al. 2004), nous expliquons qu'elles en sont les conséquences pour les praticiens du marketing.

\section{La difficulté à rapprocher les conditions d'expérience des conditions de la vie quotidienne}

Alors que, dans la vie quotidienne, les effets d'un message de communication commerciale se font ressentir plusieurs jours après (par exemple lorsque l'individu est dans le magasin), la recherche académique, utilisant la méthode expérimentale, mesure les effets quelques instants après l'exposition. Dès lors, l'oubli de la source et de l'exposition ne s'effectue pas comme dans la vie quotidienne. Par ailleurs, l'attention des individus, en laboratoire, est souvent focalisée sur les messages publicitaires. Par conséquent, la plupart des recherches académiques n'ont pu étudier les effets des messages vus rapidement avec une faible attention et rapidement oubliés. Dans la mesure où cette situation est fréquente dans la vie quotidienne, nous expliquons comment les contextes théorique et méthodologique de la cognition implicite nous ont permis de construire une procédure expérimentale plus proche de la réalité des consommateurs.

\section{Problème méthodologique}

L'immense majorité des recherches en communication persuasive, dans le domaine commercial ou politique, ont fondé leurs modèles sur des méthodes qui enregistrent et mesurent les réactions des individus au message après l'exposition (Petty et Cacioppo, 1986). À partir de ces effets attitudinaux, les chercheurs essayent, par inférence, effectuée a posteriori, de modéliser des traitements cognitifs et affectifs qui se sont déroulés pendant l'exposition. En mobilisant les récentes recherches en psychologie cognitive et linguistique et à l'aide d'un logiciel d'analyse de discours de dernière génération (logiciel d'analyse cognitivo-discursive Tropes), nous expliquons comment nous avons pu contourner ce problème méthodologique en créant une procédure d'enregistrement 
“ en temps réel ${ }^{1}$ ” des réponses cognitives à un message de communication politique persuasive à la télévision (méthode “ECER”, Fourquet-Courbet et Courbet, 2004). La méthode, présentée dans la troisième partie, permet notamment de relier de manière valide les réponses cognitives du récepteur aux éléments du message qui les ont déclenchées. Les résultats des premières expériences appliquant la méthode ECER montrent que les individus ne traitent pas les messages selon les deux routes, centrale et périphérique, modélisées dans les théories à double-processus de Petty et Cacioppo (1986) et Chaiken $(1987)^{2}$. Nous discuterons systématiquement les implications théoriques et managériales de ces nouvelles recherches.

\section{1 - Expliquer le lien attitude-comportement : le modèle MODE}

Le modèle MODE signifie littéralement "la motivation et l'opportunité comme déterminants du processus qui relie l'attitude au comportement" (Motivation and Opportunity as DEterminants ; Schuette et Fazio, 1995). Il postule que les attitudes influencent le comportement à travers deux types de processus qui peuvent opérer seul ou en interaction. Le premier processus est appelé "spontané" car il repose sur une activation automatique et non consciente des attitudes préexistantes. Il opère essentiellement lorsque les comportements sont rapides et peu réfléchis. C'est le cas de la plupart des achats de consommation courante. Le second processus, dit " délibéré", nécessite une certaine force motivationnelle, du temps, des ressources attentionnelles et cognitives pour traiter les informations disponibles. Les processus délibérés impliquent des traitements qui consomment des efforts. Souvent, l'individu examine en détail le rapport coût-bénéfice en analysant l'utilité du futur comportement. Dans un contexte de consommation, on retrouve cette situation lorsque le comportement est fortement impliquant (achats importants). On retrouve également ce cas en marketing et communication politiques avec l'acte de vote.

Même si, pour des raisons pratiques, nous les présentons de manière séparée, ces traitements sont très rarement purement spontanés ou purement délibérés. Il y a, premièrement, un continuum entre ces deux possibilités. Deuxièmement, dans la grande majorité des comportements, ces processus sont mixés. Le lien entre attitudes et comportement implique, dès lors, des composantes automatiques et contrôlées.

\section{1 - Le traitement spontané et automatique ${ }^{3}$}

\footnotetext{
${ }^{1}$ Dans la même logique d'étude des processus “ en temps réel ", Woltma Elpers, Mukherjee et Hoyer (2004) ont récemment proposé une analyse " moment par moment" des réactions émotionnelles à des messages publicitaires humoristiques à la télévision.

${ }^{2}$ Dans la suite de l'article, nous appelons ces deux théories «théories ou modèles à double processus ». Déjà bien développés dans la littérature francophone (voir Courbet et Fourquet, 2003), nous ne les expliquons que succinctement.

${ }^{3}$ Les processus automatiques sont, en psychologie cognitive, des processus sans charge mentale, qui ne consomment pas d'attention et qui ne peuvent être intentionnellement contrôlés. Au contraire des traitements contrôlés, ils ne sont donc pas conscients (pour une synthèse, voir Bargh et Chartrand, 1999).
} 
Dans le cas d'un traitement spontané, les attitudes préexistantes peuvent, d'une part, influencer les perceptions de l'individu et, d'autre part, avoir un impact direct sur son comportement, sans que l'individu ait un quelconque raisonnement conscient. C'est le cas notamment quand une attitude à l'égard d'un produit est très accessible en mémoire. Dès lors, celle-ci peut être activée automatiquement quand l'individu rencontre l'objet d'attitude. Lorsque l'achat est rapide, devant le linéaire, l'individu serait immédiatement attiré ( $v s$. non attiré) par le produit en fonction de la valence de l'attitude. En effet, une attitude fortement accessible conduit automatiquement à avoir des perceptions immédiates et à émettre, très rapidement, des jugements cohérents avec elle. Sur le plan méthodologique, ce sont les temps de réponse aux questions attitudinales qui permettent de mesurer l'accessibilité de l'attitude (Courbet, 2003).

Dans un contexte de consommation, Fazio, Powell et Williams (1989) ont montré que l'accessibilité de l'attitude à l'égard d'un produit peu impliquant est un indicateur prédictif du comportement. Dans le cadre du marketing et de la communication politiques, des recherches ont également montré que plus l'attitude à l'égard d'un homme politique est accessible en mémoire et plus celle-ci est prédictive des comportements. Fazio et Williams (1986) ont interrogé des électeurs en trois temps. Ils leur ont d'abord demandé d'exprimer leur degré d'accord avec la phase “ un bon président pour les quatre prochaines années pourrait être Ronald Reagan" (le temps de réponse a alors été mesuré). Dans une seconde phase, quelques semaines plus tard, après un débat entre Reagan et son concurrent, on a demandé aux mêmes individus d'en donner leur perception. Enfin, au lendemain de l'élection, ils ont précisé pour qui ils ont voté. Il apparaît que les sujets ayant les attitudes les plus accessibles (c'est-à-dire qui avaient répondu le plus rapidement lors de la première phase) étaient plus consistants dans leurs réponses que ceux qui avaient les attitudes les moins accessibles (c'est-à-dire ceux ayant répondu le moins rapidement). Non seulement ceux qui ont une attitude fortement accessible ont pensé que leur candidat avait été le meilleur lors du débat, mais, de plus, leur vote était plus conforme à leur attitude de départ. Le lien attitude-comportement semble donc être d'autant plus fort que les attitudes sont accessibles en mémoire.

\section{2 - Le traitement délibéré}

Le seul processus spontané est peu influent quand le récepteur est très motivé et capable de contrôler ses réponses verbales et/ou comportementales. En effet, dès lors, c'est un traitement dit délibéré qui se déclenche. Alors que le modèle du processus spontané insiste sur des attitudes préexistantes et leur accessibilité, dans le modèle du processus délibéré, l'individu ne se fonde pas sur une attitude préexistante mais sur les données de l'environnement. Ce processus est donc caractérisé par un important travail cognitif ; il implique l'examen minutieux de l'information disponible mais aussi l'évaluation des conséquences potentielles de l'engagement dans un comportement particulier. Ces évaluations détermineraient l'intention comportementale (Fazio et Towles-Schwen, 1999). 
La version récente de la théorie du comportement planifié, appliquée à la communication persuasive (Ajzen, 2001), est un type de traitement délibéré liant les attitudes aux comportements. Selon ce modèle, un grand nombre de conduites sociales sont issues d'un contrôle volontaire de la part d'un individu. Il existerait une "chaîne causale” aboutissant au comportement observé. Le principal déterminant serait l'intention comportementale, elle-même fondée sur deux ensembles de croyances : l'attitude à l'égard du comportement et deux types de normes subjectives (les normes injonctives qui sont dans le message- et les normes descriptives - “ ce que je crois que les autres font ”-). Dans sa dernière version, le modèle tient par ailleurs compte de facteurs exogènes qui peuvent agir sur la chaîne causale et exercer un effet indirect sur l'intention comportementale et la conduite effective du sujet. Ajzen a introduit dans son modèle la fréquence avec laquelle le comportement a été réalisé dans le passé (e.g. l'habitude d'achat) ainsi que le concept de contrôle perçu du comportement : un comportement dépendrait également de la perception de facilité ou de difficulté à le réaliser qu'en a l'individu (issue notamment de ses expériences passées avec ce type de comportement). Dès lors, la conduite peut échapper au contrôle de l'individu.

Comme indiqué dans le nom du modèle MODE, le déclenchement de l'un ou l'autre de ces traitements est déterminé par la motivation et l'opportunité de l'individu à délibérer. La motivation est une force qui va inciter l'individu à s'engager dans un raisonnement délibéré requérant un grand effort cognitif et de la réflexion. Elle est également fonction de l'importance perçue des conséquences que pourrait avoir pour l'individu une erreur de jugement. En effet, s'ils craignent d'aboutir à une conclusion non valide, les individus tendront à s'engager dans une réflexion attentive et soignée (Kruglanski, et Freund, 1983 ; Kruglanski et Webster, 1996). Mais si, comme on l'a vu, la motivation est liée à une recherche de précision et d'exactitude, elle peut aussi être liée à des objectifs plus spécifiques de présentation sociale. Dans tous les cas, c'est la perception d'un coût potentiel qui va motiver à davantage délibérer. La motivation à s'engager dans un traitement délibéré n'est pas suffisante : l'individu doit également avoir l'opportunité de s'engager dans un tel traitement, c'est-à-dire disposer de temps et de ressources cognitives.

Des recherches sur le rôle de chaque déterminant sont intéressantes pour les praticiens, notamment pour la conception de campagnes de communication mettant en avant les caractéristiques d'un point de vente. Dans une expérience, Sanbonmatsu et Fazio (1990) ont présenté à des sujets des évaluations contradictoires de magasins. On leur a donné une information globale négative sur un magasin nommé Brown mais une information spécifique positive sur son rayon photo. On leur a donné une information globale positive sur un autre magasin nommé Smith mais une information spécifique négative sur son rayon photo. Les sujets devaient ensuite dire où ils achèteraient un appareil photo. Ceux qui ont choisi Smith se sont basés sur leur évaluation générale et ont eu recours à un traitement spontané et sans effort pour arriver à cette décision. A l'inverse, ceux qui ont choisi Brown se sont engagés dans un traitement demandant un effort pour récupérer en mémoire l'information relative aux attributs spécifiques de chaque magasin. La motivation et l'opportunité 
ont également été manipulées. On a, d'une part, augmenté la motivation de la moitié des participants en leur précisant que leur choix serait comparé à ceux des autres personnes et qu'ils devraient l'expliquer publiquement. On a, d'autre part, manipulé l'opportunité en introduisant une pression temporelle chez la moitié des participants qui devaient décider en 15 secondes maximum. Il apparaît que le choix de Brown, fondé sur le traitement délibéré d'informations spécifiques, est fait par des sujets très motivés (ayant peur de faire une erreur de jugement) et ayant une forte opportunité (ayant le temps de faire leur choix). En cas de faibles motivation et opportunité, les participants n'ont a priori pas pu extraire l'information spécifique en mémoire et se sont contentés de choisir en fonction de leur attitude globale.

\section{2) Les effets de la publicité oubliée : les apports de la socio-cognition implicite}

Les concepts et méthodes issus des récentes recherches dans le domaine de la socio-cognition implicite semblent pertinents pour contribuer à modéliser les effets automatiques de la communication persuasive et de la publicité. Après avoir indiqué quelques apports théoriques issus de ce domaine, nous donnons les résultats d'une recherche que nous avons réalisée sur l'influence de la publicité vue rapidement et oubliée.

\section{1 - Les influences sur les attitudes explicite et implicite}

En suivant le récent modèle de la double attitude, issu de la psychologie sociale cognitive (Wilson et al. 2000), on pourrait distinguer deux voies d'influence conduisant à former deux types d'attitudes : l'attitude explicite et l'attitude implicite. L'attitude explicite correspond au concept d'attitude tel qu'il a été classiquement défini en psychologie sociale et dans les études en communication persuasive : l'attitude est une disposition interne qui sous-tend les réponses favorables ou défavorables à un objet. Mesurable par des échelles, elle est constituée d'une composante cognitive relative aux attributs de l'objet, d'une évaluation affective unidimensionnelle de l'objet et d'une composante conative relative à l'intention d'action vis-à-vis de l'objet (Eagly et Chaiken, 1993). Alors que la personne est capable d'expliquer les véritables déterminants de son attitude explicite, elle est dans l'impossibilité de verbaliser ceux de son attitude implicite. Ainsi, en approfondissant la définition de Greenwald et Banaji (1995) : les attitudes implicites sont des traces de l'expérience passée, ni identifiées ni attribuées par introspection, qui polarisent affectivement et médiatisent les pensées, sentiments et jugements présents relatifs à des objets sociaux. Activées automatiquement, elles sont un puissant déterminant du comportement lorsqu'il est faiblement impliquant. Issues de la mémoire implicite, les attitudes implicites ne sont mesurables que par des techniques indirectes comme l'étude des temps de réponse, les choix forcés ou des techniques qui font travailler en opposition attitude implicite et attitude explicite (Greenwald et al. 1998). 
Même si le débat sur les liens qui unissent les deux attitudes n'est pas encore tranché (Fazio et Olson, 2003), Wilson et al. (2000) ont suggéré que, chez une même personne, attitude explicite et attitude implicite à l'égard d'un même objet social sont toutes deux stockées en mémoire. Quand on demande à une personne d'évaluer très rapidement un objet connu, l'attitude implicite est automatiquement activée en mémoire. La personne émet alors un jugement qui est souvent congruent avec l'attitude implicite. Si la personne prend davantage de temps pour juger cet objet et si une attitude explicite sur l'objet est déjà engrammée, c'est alors cette dernière qui est intentionnellement (consciemment) récupérée. Cette récupération intentionnelle et contrôlée prend davantage de temps que la récupération automatique (non consciente).

Dans la vie quotidienne, le temps mis pour traiter et juger un objet social et le niveau d'élaboration dépendent des ressources cognitives et attentionnelles allouées au traitement des informations. Afin de faciliter la présentation, nous divisons le continuum de ressources cognitives allouées au traitement des messages persuasifs en deux parties. On obtient ainsi deux grands types de processus ou deux voies d'influence :

- une voie dans laquelle les messages sont traités avec une plus forte allocation d'attention. Cette voie conduit directement à former, changer ou renforcer l'attitude explicite. La décision comportementale est réfléchie et le comportement planifié à l'avance ;

- une voie dans laquelle les messages sont traités avec une plus faible allocation d'attention. Cette voie n'influence pas directement l'attitude explicite mais avant tout l'attitude implicite. A la différence de la "voie d'influence explicite”, les principaux processus médiatisant la persuasion opèrent essentiellement pendant la phase où la personne est amenée à récupérer les traces mnésiques et à prendre une décision comportementale. Ainsi, le comportement n'est pas planifié et la décision comportementale se prend juste avant la conduite (par exemple, au point de vente). Ce modèle a constitué le contexte théorique pour l'expérience suivante.

\section{2 - Les influences de la publicité interstitielle vue rapidement sur Internet et oubliée}

Internet se prête parfaitement à l'étude de la publicité vue rapidement et aussitôt oubliée. L'internaute ne porte généralement qu'une faible attention aux publicités interstitielles, considérées comme de l'information non pertinente pour sa tâche principale (Drèze et Zufryden, 2000). Dans le message, la narration publicitaire est simple, le logotype de la marque occupe souvent un espace important. Les études publicitaires sur Internet, effectuées par des organismes rigoureux ${ }^{4}$, montrent que, de manière générale, lorsqu'un individu est en contact avec une publicité sur Internet, que ce soit un bandeau (bannière) publicitaire ou une publicité interstitielle, l'attention qu'il alloue au message est trop faible pour qu'il puisse élaborer une attitude explicite à l'égard de la marque à la

\footnotetext{
${ }^{4}$ TNS Media Intelligence, Ipsos Médias.
} 
suite de quelques expositions. Le message, s'il est vu et traité, est aussitôt oublié. Quels sont les mécanismes d'influence de telles publicités?

Pour l'illustration, partons du principe qu'une personne a été en contact avec une ou plusieurs publicités interstitielles sur Internet pour un nouveau produit de grande consommation : une pizza emballée de marque "Lomis". Nous émettons l'hypothèse que le principal mode d'influence suit le processus suivant (nous simplifions pour l'illustration) : parce que la pizza n'est pas un produit de forte implication et parce que le média Internet n'incite pas à focaliser une forte attention sur les publicités interstitielles, la personne ne traite pas le contenu argumentaire de façon détaillée. Elle voit rapidement ce nom de marque et va l'oublier quasi immédiatement. Mais sa mémoire implicite (non consciente) engrammera le logotype et des traces publicitaires sans que la personne en soit consciente. Si, plus tard, cette personne veut acheter une pizza dans un hypermarché, elle va être en contact avec la marque "Lomis" dans le rayon des produits frais. Le peu de temps généralement accordé pour les achats en grande surface, la différence de contexte ainsi que le long délai entre l'exposition publicitaire sur Internet et la vision de la marque dans les rayons du magasin ne conduisent pas le consommateur à se souvenir explicitement du message. Il va être amené à juger différentes marques en concurrence dans le rayon, notamment la marque Lomis, afin de prendre une décision d'achat. La personne juge les marques très rapidement (elle prend peu de temps pour faire ses courses, elle fait peu d'efforts cognitifs). Ce sont les traces présentes en mémoire implicite qui vont être utilisées pour former une attitude implicite (Bargh et Apsley, 2001). Celle-ci va être automatiquement activée à la vision de la marque "Lomis". Face au produit et parce que la marque est déjà mémorisée, la perception et la reconnaissance sont plus rapides : c'est un effet de " fluidité perceptive ". Dans un grand nombre de cas, ces traces mnésiques peuvent même être associées à des affects positifs ou négatifs (Lee et Labroo, 2004). Dans ce contexte, c'est l'attitude implicite qui va être considérée par l'individu pour formuler l'intention d'achat. En effet, au regard des travaux sur le lien entre attitude implicite et comportement (Fazio et Towles-Schwen, 1999), il y a une forte probabilité pour que, dans certaines circonstances à définir, l'intention d'achat de la marque soit formée à partir de l'attitude implicite à l'égard de la marque. Celle-ci serait prédictive du comportement d'achat, si, sur le lieu de vente, l'environnement n'est pas ouvertement défavorable à cet acte.

Nous avons émis l'hypothèse qu'une publicité pour une nouvelle marque vue rapidement (pendant 3 secondes) et aussitôt oubliée, laisse des traces mnésiques qui seront, une semaine après, favorables aux jugement que l'individu porte sur la marque (Courbet et al., 2004). Même si le consommateur est incapable de rappeler ou de reconnaitre la marque comme étant une marque déjà vue préalablement, nous pensons que, 8 jours après, il va tout de même davantage l'apprécier. L'objectif est de montrer que la publicité sur Internet a des effets sur l'attitude implicite à l'égard de la marque qui tiennent dans le temps et, sans doute, en accord avec le modèle MODE, sur le comportement d'achat. Les méthodes actuellement utilisées pour enregistrer des représentations et 
des jugements à l'égard de la marque (les échelles et les questionnaires écrits) ne permettent pas d'avoir accès à l'attitude implicite. Les techniques utilisant le langage permettent en effet d'accéder à l'attitude explicite et aux réponses verbales mais pas à l'attitude implicite. L'analyse des temps de réponse (TR) peut mettre en évidence des traces mnésiques de l'exposition publicitaire là où les techniques de mesure classiques ne montrent pas d'effet. La méthode d'enregistrement des TR est intéressante ici pour trois raisons. D'une part, elle est tout à fait adaptée pour étudier les propriétés de base de la cognition implicite (Greenwald et Banaji, 1995). D'autre part, au stade actuel des recherches en psychologie, le TR est le meilleur indicateur de l'attitude implicite (Fazio, 2000). Enfin, le temps de réponse, et donc l'attitude implicite, est le plus puissant déterminant du comportement lorsque ce dernier est faiblement impliquant (Schuette et Fazio, 1995), ce qui est le cas des comportements dans notre domaine appliqué : plus les TR sont courts et plus les évaluations seraient prédictives de l'achat. C'est ce temps de réponse qui me permettra de mettre en évidence des effets de la publicité sur l'attitude implicite.

\section{3 - Elaboration d'une nouvelle méthode d'étude}

Afin de concevoir une nouvelle méthode d'étude, nous construisons une situation expérimentale ${ }^{5}$ qui se rapprocherait de la situation que nous avons décrite ci-dessus. On réalise un site Internet " de qualité professionnelle " nommé "Santé.com " et destiné, avant tout, aux étudiants. En surfant sur ce site, l'individu est exposé quatre fois à la même publicité interstitielle pour la marque "Lomis " apparaissant, à chaque fois, pendant une durée de 3 secondes. Cette marque est inédite et spécifiquement fabriquée pour l'expérience. Elle est constituée d'un nom “ Lomis ” et d'un logotype inédits. L'esthétique de ce dernier a été particulièrement travaillée pour qu'il soit jugé positivement par la plupart des personnes interrogées (afin d'obtenir une attitude positive à l'égard de cette nouvelle marque). On conçoit deux publicités interstitielles différentes (publicités A et B). Toutes les deux ont les mêmes dimensions et sont signées par "Lomis ". Le contenu publicitaire est cependant différent dans les publicités A et B. On conçoit une première publicité interstitielle A constituée du seul logotype de la marque. Dans la publicité B, le logotype Lomis est couplé au mot "pizza”.

En suivant le plan expérimental 2 (exposés vs. non exposés aux publicités) X 2 (types de contenu publicitaire : marque seule vs. marque et mot), les sujets sont répartis au hasard entre les trois groupes suivants :

- Groupe “marque seule" : vision du site avec 4 expositions de 3 secondes à la publicité contenant la marque seule ;

- Groupe " marque + mot" : vision du site avec 4 expositions de 3 secondes à la publicité contenant la marque associée au mot "pizza" ;

\footnotetext{
${ }^{5}$ Pour faciliter la compréhension, nous ne décrivons ici qu'une partie de la recherche expérimentale menée.
} 
- Groupe contrôle (GC) : il ne voit aucune marque mais répond uniquement au questionnaire de la deuxième phase.

La procédure comprend trois phases :

- Première phase : On demande aux sujets de consulter un site Internet relatif à l'alimentation et la santé puis ils répondent à un court questionnaire écrit où ils donnent leur avis sur la qualité et l'utilité d'un tel site pour améliorer les habitudes alimentaires des étudiants.

- Deuxième phase : sans que les sujets ne fassent de lien avec la première phase, une semaine après, d'autres expérimentateurs reconvoquent les mêmes personnes afin de réaliser une enquête pour des entreprises. On dit que celles-ci souhaitent sortir de nouveaux produits et désirent tester auparavant la pertinence de leur nom de marque et de leur logotype afin de voir s'ils sont bien évalués et bien adaptés au secteur de produits commercialisés. On demande aux sujets de répondre à un questionnaire passé par ordinateur et portant sur quatre nouvelles marques : Lomis et trois autres marques fillers (remplisseurs).

- Troisième phase : immédiatement après, on demande aux personnes de répondre par écrit et de dire franchement si elles ont déjà vu une des marques que l'on vient de leur montrer. On supprime les réponses des sujets ayant reconnu la marque Lomis comme étant celle vue une semaine auparavant.

On construit donc un cadre expérimental où on ne garde que les données issues des sujets qui sont incapables de se souvenir d'avoir vu précédemment la marque expérimentale et qui ne la reconnaissent pas, avec ou sans indice lié au contexte d'exposition. Ainsi, les critères établis en psychologie sociale (Greenwald et Banaji, 1995) pour affirmer que l'attitude est implicite sont remplis. Cependant, comme dans la littérature, la définition du concept d'attitude implicite demeure souvent floue, précisons comment nous opérationalisons les concepts. Tous les jugements et attitudes émis par les groupes expérimentaux sont des jugements et attitudes implicites, dans la mesure où personne, au moment de répondre, ne s'est souvenu de la marque, de la publicité et de la phase d'exposition. Tous les jugements émis par le groupe contrôle sont des jugements et attitudes explicites. Les jugements et attitudes implicites (pour les groupes expérimentaux) ou explicites (pour le groupe contrôle) sont opérationalisés par deux types de mesures. Premièrement, par la mesure de réponses verbales que le sujet émet sur la marque. Deuxièmement, par la mesure du temps que le sujet met pour donner sa réponse (mesure du TR). L'enregistrement des variables dépendantes s'effectue grâce à un programme informatisé qui déroule le questionnaire automatiquement. Le sujet doit répondre à la fois le plus justement et le plus rapidement possible à différentes questions relatives à la marque.

Les résultats montrent que la méthode de l'analyse des TR a effectivement permis de mettre en évidence des traces mnésiques implicites de la marque vue au sein de publicité sur Internet, une 
semaine auparavant, alors que l'analyse des réponses verbales n'a rien pu montrer (voir tableau 1). Le temps mis pour répondre “oui” est plus court dans les groupes exposés que dans le groupe contrôle.

Tableau 1 : Réponses verbales et temps de réponse " oui " à l'item

“J'apprécie cette nouvelle marque " (extrait de Courbet et al. 2004)

\begin{tabular}{|c|c|c|c|}
\hline & $\begin{array}{c}\text { Exposés à la } \\
\text { marque } \\
\text { seule }\end{array}$ & $\begin{array}{c}\text { Exposés à } \\
\text { marque } \\
\text { texte }\end{array}$ & $\begin{array}{c}\text { Groupe } \\
\text { contrôle (non } \\
\text { exposé) }\end{array}$ \\
\hline $\begin{array}{c}\text { Réponses } \\
\text { verbales }\end{array}$ & $\mathbf{n}$ & $\mathbf{n}$ & $\mathbf{n}$ \\
\hline Oui & 38 & 32 & 42 \\
\hline Non & 22 & 24 & 24 \\
\hline TR (en ms) & $\mathbf{M}$ & $\mathbf{M}$ & $\mathbf{M}$ \\
\hline TR oui & $1667^{*}$ & $1681^{*}$ & 2023 \\
\hline
\end{tabular}

Notes $: \mathrm{n}=$ nombre de sujets $; \mathrm{TR}=$ Temps de réponse $; \mathrm{M}=$ moyenne en $\mathrm{ms} ; *$ les comparaisons avec le groupe contrôle effectuées par tests $t$ de Student sont significatives à $p<.05$. Des tests du Khi carré montrent qu'il n’y a pas de différences significatives entre les réponses verbales des trois groupes.

Ainsi, la méthode élaborée permet d'étudier des processus de réception et d'influence non conscients de la publicité sur Internet et, plus particulièrement la présence de traces affectives associées de manière implicite avec la marque. Les marques et les expositions publicitaires de la semaine précédente, bien que toutes deux oubliées, ont laissé des traces en mémoire sans que les sujets en aient conscience. Les messages publicitaires ont agit sur l'attitude implicite. Ces traces mnésiques implicites ne sont pas mises en évidence par les mesures verbales d'attitude mais par la méthode des temps de réponse. Quand les sujets préalablement exposés ont vu la marque expérimentale, l'attitude implicite s'est immédiatement et automatiquement activée. En demandant de répondre rapidement à une question de nature affective, la réponse, ne nécessitant pas une forte allocation de ressources cognitives, est basée sur l'attitude implicite et est congruente à elle (Lee et Labroo, 2004).

Cette étude a également des apports opérationnels puisqu'elle montre clairement que les mesures d'efficacité publicitaire utilisées par les praticiens et reposant soit sur le taux de clic, soit sur des enquêtes vu/lu, sous estiment largement l'influence. En effet, ce n'est pas parce que l'internaute ne clique pas sur le bandeau que ce dernier n'a pas une influence. Une bannière qui n'est ni lue, ni rappelée dans les tests peut avoir influencé l'attitude implicite.

\section{4 - Nouvelles perspectives ouvertes}

$\mathrm{Si}$, comme nous l'avons montré dans notre cas, le récepteur n'a pas conscience des mécanismes d'influence et de ce qu'il mémorise, quelles sont les validités et les limites des méthodes reposant sur le langage et donc des théories qu'elles élaborent? Les théories de la socio-cognition implicite offrent la possibilité de mieux connaître les rôles respectifs des processus conscients et non 
conscients dans les traitements psychologiques des messages médiatiques et dans leur processus d'influence. Elles permettraient de mieux savoir comment opèrent les traitements des éléments et des dispositifs sémiotiques et iconiques, esthétiques, linguistiques, propres aux systèmes multimédias (clignotement d'éléments, apparition brève des marques, liens hypermédia...). A l'avenir, il s'agirait d'expliquer comment se déroulent les différentes phases du processus d'influence médiatique depuis le premier contact avec des éléments du message, leur perception, les processus attentionnels mis en œuvre, la compréhension, les réactions émotionnelles, les processus de décision, de jugement et de formation ou de changement d'attitude (immédiats ou différés), la mémorisation. Ces théories permettent également d'étudier le rôle des traces mnésiques conscientes et non conscientes dans la décision comportementale ou le futur comportement d'achat.

La méthode utilisée donne la possibilité d'étudier de manière analytique les rôles de plusieurs facteurs en interaction que l'on retrouve dans la vie quotidienne. Premièrement, le rôle des facteurs personnels (l'implication dans la catégorie de produits) ; deuxièmement, le rôle des facteurs propres aux contextes socio-physico-temporel de réception (rôle d'éléments distracteurs à proximité de l'internaute) ; troisièmement, le rôle du contexte médiatique entourant l'apparition de la publicité (page web qui génère des émotions positives).

La méthode peut s'appliquer à d'autres médias où la publicité et, plus généralement, les messages de communication persuasive, sont traités par les récepteurs avec une faible attention (télévision, radio, presse ou affichage). Enfin, il s'agit à l'avenir de se pencher sur le rôle crucial de l'attitude implicite et de l'attitude explicite dans le comportement que souhaite instaurer la communication persuasive. Quand on sait que plus le sujet répond vite et plus sa réponse est prédictive de son comportement (Fazio et Towles-Schwen, 1999), on voit les perspectives qu'offre la méthode d'enregistrement des temps de réponse utilisée dans un contexte de réception et d'influence de la publicité, mais également dans d'autres domaines de la communication médiatique comme la santé publique ou la politique.

Il est probable que ces processus, mis en route en situation de faible allocation d'attention, influencent d'autant plus le comportement d'achat que la personne ne se souvient pas d'avoir été exposée à des publicités pour la marque. Si, pendant l'acte d'achat, les consommateurs pensent qu'ils ont déjà été exposés, l'attitude implicite pourrait être inhibée (Courbet, 2003). C'est l'attitude explicite qui prendrait dès lors le dessus.

\section{3) Etudier les processus cognitifs " en temps réel" : les apports de la psychologie cognitive et linguistique}

Nous présentons une nouvelle méthode, appelée méthode d'Etude des Cognitions En Réception (ECER, Fourquet-Courbet et Courbet, 2004), qui, en analysant « en temps réel » les principaux processus cognitifs, pourrait dépasser certaines limites auxquelles se heurtent les recherches portant 
sur la voie de l'influence explicite. Nous présentons les principales caractéristiques de la méthode. Nous expliquerons ensuite comment, en l'appliquant au sein d'un plan expérimental, elle a permis de porter un nouveau regard sur le rôle de l'implication dans les traitements cognitifs du récepteur.

\section{1 - Les principales caractéristiques de la méthode ECER}

Un accès aux pensées du récepteur « en temps réel »

Les verbalisations du sujet pendant la réception du message sont considérées comme des observables à partir desquels on peut connaître son activité mentale (Caverni, 1988) : elles sont le résultat d'un traitement contrôlé du sujet et rendent compte de ses réponses cognitives en mémoire de travail. La méthode ECER permet d'accéder aux réponses cognitives du récepteur et de les recueillir en temps réel, pendant l'exposition au message. On accède ainsi à la dynamique des traitements : les processus cognitifs ne sont pas inférés a posteriori comme dans la " tâche de listage des pensées" (Greenwald, 1968). Dans cette dernière, on demande aux sujets de dire, une fois que l'exposition est terminée, ce à quoi ils ont pensé pendant qu'ils étaient face au message. La méthode ECER est particulièrement intéressante pour les messages télévisés de plusieurs minutes (e.g. discours d'un homme politique) susceptibles de provoquer, tout au long du message, de nombreuses réponses cognitives, contre-argumentations...

Lors de la phase de recueil, le sujet regarde un message télévisé et dit «ses pensées » à voix haute. Un dispositif d'enregistrement audio discret ${ }^{6}$ permet d'enregistrer à la fois le message diffusé et les pensées verbalisées des sujets : on pourra donc retranscrire les verbalisations concomitantes en faisant un lien avec les différents éléments et indices figurant dans le message télévisé, préalablement codé.

\section{Une définition plus opérationnelle de l'élaboration cognitive}

En recueillant ainsi les verbalisations, la méthode ECER permet non seulement d'analyser en temps réel les processus, mais également d'étudier l'élaboration cognitive. La définition de l'élaboration cognitive donnée par Petty et Cacioppo (1986, p.128, “l'importance avec laquelle une personne pense aux arguments contenus dans un message") étant relativement floue ${ }^{7}$, nous avons, tout d'abord, opérationnalisé ce concept. Grâce au double enregistrement, on peut directement relier les réponses cognitives du sujet à leurs déclencheurs figurant dans le message. Par ailleurs, on peut évaluer le nombre de verbalisations pendant la réception (ce qui n'était pas le cas avec un recueil $a$ posteriori). Ainsi, on analyse les traitements sous deux angles distincts :

\footnotetext{
${ }^{6}$ L'individu sait qu'il est enregistré mais ne voit pas le dispositif.

${ }^{7}$ Les deux principaux modèles de persuasion à double processus (Petty et Cacioppo, 1986 et Chaiken, 1987) considèrent, d'une part, qu'un indice périphérique est systématiquement traité avec une faible élaboration et, d'autre part, qu'un indice central (lié au contenu du message) est systématiquement traité avec une forte élaboration. Ils n'envisagent donc pas, par exemple, la possibilité de fortement élaborer un indice périphérique comme la manière de s'habiller d'un homme politique. Nos résultats, développés plus loin,
} 
- l'identification de la "nature" du traitement en se fondant sur les indices qui ont déclenché les réponses cognitives. Indépendamment de l'effort cognitif fourni, si le traitement est déclenché par un indice non lié à l'argumentation (e.g. la musique d'une publicité pour une voiture), il sera dit "périphérique". A l'inverse, s'il est déclenché par un argument du message (e.g. les caractéristiques techniques de la voiture), il sera dit "central" ;

- l'évaluation de la "force" du traitement, c'est-à-dire son niveau d'élaboration : si le nombre de pensées déclenchées lors de la réception est élevé, le traitement sera dit "fortement élaboré". A l'inverse, si le nombre de pensées déclenchées lors de la réception est faible, le traitement sera dit "faiblement élaboré".

\section{Analyse de l'activité cognitive du récepteur}

Une fois les verbalisations recueillies, nous analysons les discours des récepteurs avec l'analyse cognitivo-discursive (ACD, Ghiglione, Kekenbosch et Landré, 1995). Techniquement, c'est le logiciel Tropes qui automatise cette analyse : on objective ainsi le traitement des données tout en augmentant les validités et fidélités de l'analyse. Plusieurs catégories de variables permettent d'étudier les traitements cognitifs des récepteurs. D'une part, avec une analyse lexicométrique, on compte les marqueurs discursifs comme les styles de verbes, les joncteurs ou connecteurs (e. g. conjonctions de coordination et de subordination), les modalisations (adverbes ou locutions adverbiales permettant au locuteur de préciser son discours), les types d'adjectifs, les pronoms personnels, relatifs et possessifs, les modes des verbes, les déterminants.

Lorsqu'un individu traite un message, il se fixe des buts à partir desquels il construit des stratégies cognitivo-discursives (Ghiglione et Trognon, 1993). Celles-ci sont caractérisées par des marqueurs discursifs particuliers, observables dans les réponses cognitives qu'il émet. Nous utilisons donc ces marqueurs pour étudier le rapport que le sujet entretient avec «le monde mis en scène » dans le message (voir Fourquet-Courbet et Courbet, 2004). En étudiant les référents centraux des réponses cognitives (c'est-à-dire les thèmes contenus dans les réponses cognitives), on effectue une analyse des «références mises en scène » par le sujet. La méthode ECER a été testée dans le cadre d'une expérimentation sur le rôle de l'implication dans les traitements cognitifs. Nous avons étudié l'impact de l'implication sur la nature et le niveau d'élaboration des traitements des sujets.

\section{2 - Premiers résultats : une meilleure compréhension du rôle de l'implication dans les traitements cognitifs du récepteur}

\section{Présentation du dispositif expérimental}

Deux groupes de sujets, l'un impliqué et l'autre non impliqué, ont été exposés à un message de communication politique télévisée construit pour l'expérience (103 sujets ont participé à 
l'expérience). Le film, d'une dizaine de minutes, met en scène un homme politique annonçant des mesures de restriction de circulation dans la ville et autour de la faculté où les sujets font leurs études. Nous y avons mis de nombreux indices périphériques susceptibles d'attirer l'attention du récepteur et de provoquer un traitement de nature périphérique (e.g. flash d'appareils photo, tics verbaux de l'homme politique, vêtements incongrus, mimiques faciales accentuées,... $)^{8}$. L'implication a été créée en manipulant la date de mise en œuvre des mesures annoncées. Dans un groupe, les mesures sont très dérangeantes puisque l'homme politique annonce qu'elles seront mises en place dans un délai de trois mois (condition de forte implication vs. dans 7 ans dans la condition de faible implication ${ }^{9}$ ).

Principaux résultats (pour plus de détails, voir Fourquet-Courbet et Courbet, 2004).

Un traitement plus élaboré des indices centraux et périphériques chez les sujets impliqués

Dans les discours recueillis, on a procédé aux analyses suivantes :

- on détecte, pour chaque verbalisation, l'indice déclencheur (périphérique vs. central) afin de déterminer la nature du traitement. Conformément aux modèles de persuasion à double-processus (Chaiken et Trope, 1999), on s'attend, d'une part, à ce que les sujets fortement impliqués déclenchent davantage leurs verbalisations en réaction à des indices centraux et, d'autre part, à ce que les sujets faiblement impliqués réagissent davantage à des indices périphériques ;

- on compte, pour chaque sujet, le nombre de ses interventions ; on s'attend à ce que les sujets impliqués aient un traitement plus élaboré (i.e. interviennent davantage) que les sujets peu impliqués.

Les résultats montrent que, comme nous l'attendions, les sujets impliqués ont eu un traitement plus élaboré (ont émis un nombre de réponses cognitives pendant la réception plus élevé) que les sujets faiblement impliqués. Toutefois, la deuxième conclusion ouvre de nouvelles perspectives : si les sujets impliqués élaborent davantage, ils traitent autant les indices centraux que les indices périphériques du message (voir Tableau 2).

Tableau 2 : Force et nature des traitements selon le niveau d'implication

\begin{tabular}{|c|c|c|c|c|}
\hline Variables & $\begin{array}{c}\text { Groupe 1 - } \\
\text { impliqués } \\
M^{*}\end{array}$ & $\begin{array}{c}\text { Groupe } 2 \text { - non } \\
\text { impliqués } \\
\mathbf{M}^{*}\end{array}$ & $\begin{array}{c}\text { Test t de } \\
\text { Student : } \\
\text { valeur du } t\end{array}$ & $p$ \\
\hline \multicolumn{5}{|l|}{ NIVEAU D'ELABORATION } \\
\hline Nombre d'interventions & $21,9(12,50)$ & $16,7(11,54)$ & $-2,17$ & .03 \\
\hline Nombre de réponses cognitives & $20,1(12,14)$ & $14,9(10,81)$ & $-2,27$ & .02 \\
\hline \multicolumn{5}{|l|}{ DECLENCHEURS } \\
\hline Nombre de déclencheurs=indices périphé & $18,9(10,86)$ & $14,7(10,13)$ & $-2,02$ & .04 \\
\hline
\end{tabular}

\footnotetext{
${ }^{8}$ Une pré-enquête a confirmé que le matériel était crédible.

${ }^{9}$ L'implication manipulée est dite conjoncturelle, c'est-à-dire " liée à la motivation du récepteur à connaître exactement les positions de l'homme politique sur un thème pour en tirer un maximum de bénéfice ou un minimum de perte, au regard de ses intérêts personnels et matériels à court terme" (voir Fourquet-Courbet et Courbet, 2004, p.47)
} 


\section{$\underline{\text { Une structure de discours spécifique chez les sujets impliqués }}$}

Pour étudier l'impact de l'implication sur les structures de discours des sujets, nous avons :

- identifié des univers de références (les éléments sémantiques) mis en scène dans les discours des sujets. En accord avec la pragmatique de la communication (Ghiglione, 1986 ; Ghiglione et Trognon, 1993), on s'attend à ce que les sujets impliqués mettent davantage en scène les univers de références centraux du message (c'est-à-dire les mots ayant la plus forte occurrence dans le message) que les sujets faiblement impliqués ;

- compté les marqueurs discursifs utilisés par les sujets puis comparé les stratégies cognitivodiscursives développées par les sujets.

Si aucune différence n'est apparue dans les univers de références mis en scène par les sujets impliqués vs. faiblement impliqués, il apparaît que l'implication des sujets agit sur les stratégies cognitivo-discursives qu'ils développent. Les sujets impliqués ont mis en scène leur discours sur la base d'une "réalité à affirmer" et de l'évocation d'un monde réel, c'est-à-dire qu'ils ont davantage utilisé de modalisations d'intensité (qui marquent leur engagement et la fermeté de leurs positions), de verbes statifs (ancrant les référents mis en scène dans le réel) ainsi que le mode indicatif (voir tableau 3).

Tableau 3 : Marqueurs discursifs et stratégies cognitivo-discursives selon de Niveau d'implication

\begin{tabular}{|c|c|c|c|c|}
\hline Variables & $\begin{array}{c}\text { Groupe 1- } \\
\text { impliqués } \\
\mathbf{M}^{*}\end{array}$ & $\begin{array}{c}\text { Groupe } 2 \text { - non } \\
\text { impliqués } \\
\mathbf{M}^{*}\end{array}$ & $\begin{array}{c}\text { Test } t \text { de } \\
\text { Student } \\
\text { Valeur du } t\end{array}$ & $p$ \\
\hline \multicolumn{5}{|c|}{ MARQUEURS DISCURSIFS UTILISES } \\
\hline Verbes statifs & $0,08(0,02)$ & $0,07(0,03)$ & 3,18 & .001 \\
\hline Modalisations d'intensité & $0,04(0,02)$ & $0,03(0,02)$ & 2,23 & .02 \\
\hline Mode indicatif & $0,125(0,02)$ & $0,113(0,04)$ & 1,92 & .05 \\
\hline Pronom " nous" & $0,003(0,04)$ & $0,001(0,002)$ & 2,53 & .01 \\
\hline Pronom “vous" & $0,001(0,0004)$ & $0,001(0,003)$ & $-2,29$ & .02 \\
\hline Pronom " on" & $0,02(0,01)$ & $0,01(0,09)$ & 2,16 & .03 \\
\hline \multicolumn{5}{|c|}{\begin{tabular}{|l} 
STRATEGIES COGNITIVO-DISCURSIVES \\
\end{tabular}} \\
\hline Type 1: " réalité à affirmer" & $0,4(0,05)$ & $0,3(0,09)$ & 2,9 & .004 \\
\hline Type 2 : " réalité à construire " & $0,4(0,05)$ & $0,3(0,10)$ & 1,93 & .05 \\
\hline Type 3 : " réalité à justifier" & $0,06(0,02)$ & $0,06(0,04)$ & $-0,72$ & .50 \\
\hline
\end{tabular}

Note. M signifie moyenne. * Ecart type entre parenthèses

(Tableau issu de Fourquet-Courbet et Courbet, 2004)

$\underline{\text { Pas de changement d'attitude, quelles que soient la force et la nature du traitement }}$ 
Nous avons également identifié la valence (positive $v s$. négative) de chaque intervention des sujets par rapport au message ; l'hypothèse testee est que les sujets impliqués qui sont face à un message contre-attitudinal vont davantage verbaliser des pensées négatives (défavorables au message) et donc vont avoir une attitude "spécifique" à l'égard du message plus défavorable que les non impliqués. Les résultats montrent que, chez les sujets impliqués, il y avait a priori un lien entre la valence des réponses cognitives et l'attitude à l'égard du message : ce dernier étant contreattitudinal pour eux, ils ont, comme on s'y attendait, émis significativement plus de réponses cognitives négatives. Toutefois, leur attitude “spécifique " à l'égard des mesures annoncées n'est pas significativement plus négative que celle des sujets non impliqués.

Par ailleurs, les résultats ne sont pas strictement en accord avec la logique des modèles de persuasion à double-processus pour lesquels c'est le type d'indice traité qui a un impact sur l'attitude. Ici, l'attitude générale (à l'égard des mesures de restriction de circulation en général, mesurée avant et après l'exposition au message) reste stable pour les sujets impliqués et non impliqués, bien que les seconds aient eu un traitement significativement moins élaboré que les premiers et qu'ils aient traité significativement moins d'indices centraux et périphériques.

\section{3 - Discussion, perspectives théoriques et managériales}

Dans notre contexte, la méthode ECER a mis en évidence le fait qu'une forte implication incite à une plus forte élaboration des indices centraux et des indices périphériques. Les sujets fortement impliqués mettent en place des structures de discours particulières mettant en scène « une réalité à affirmer ». Au contraire de ce que planifient les modèles de persuasion à double processus, nous n'avons pas constaté de lien entre, d'une part, la force et la nature du traitement et, d'autre part, le changement d'attitude.

$\mathrm{Au}$ niveau des pratiques de marketing et de communication politiques, et si ces résultats se confirment, ce serait donc une erreur de penser que les récepteurs impliqués ne s'intéressent qu'au contenu du message. Aussi les conseillers en communication politique doivent-ils veiller à ce que les hommes politiques soignent particulièrement les éléments périphériques, parfois considérés par les politiques eux-mêmes comme des « détails ». Ces derniers ne seraient pas traités uniquement par les personnes peu intéressées par la politique ou le thème développé dans le message.

Appliquée à la communication commerciale, la méthode ECER peut être utilisée comme pré-test publicitaire. Elle permettrait de connaître en temps réel les réactions des sujets à des messages publicitaires fondées sur l'humour, l'esthétique ou encore sur des arguments techniques. On dispose d'un outil permettant de connaître les effets « en direct » de telle ou telle technique créative figurant dans un message. Dans un contexte de communication d'utilité publique (e.g. sécurité routière, campagne anti-tabac), couplée avec des mesures d'attitudes et d'intentions comportementales, la méthode ECER pourrait permettre, par exemple, de mieux concevoir des techniques d'appel à la peur (e.g. sécurité routière) pour inciter à mettre en oeuvre des comportements sécuritaires. 


\section{BIBLIOGRAPHIE}

Ajzen, I., 1991, "The theory of planned behavior", Organizational Behavior and Human Decision Processes, 50, pp.179-211

Ajzen, I., 2001, "Nature and operation of attitudes ", Annual Review of Psychology, 52, pp.27-58.

Bargh, J. A. et Chartrand T. L., 1999, The Unbearable Automaticity of Everyday Life, American Psychologist, 54, pp. 462-479.

Bargh, J. A., Apsley D.K. (eds), 2001, Unravelling the Complexities of Social Life, Washington, American Psychological Association.

Caverni, J. P., 1998, "La verbalisation comme source d'observables pour l'étude du fonctionnement cognitif", in J. P. Caverni, C. Bastien, P. Mendelsohn \& G. Tiberghien (Eds). Psychologie cognitive : modèles et méthodes. Grenoble : Presses Universitaires de Grenoble, PP.253-273.

Chaiken, S., 1987, "The heuristic model of persuasion", in M.P. Zanna, J.M. Olson, C.P. Herman (Eds.), Social influence: the ontario symposium, vol. 5, Hillsdale, NJ: Erlbaum, 1987, pp. 3-39.

Chaiken, S., Tropes Y. (eds), 1999, Dual-Process Theories in Social Psychology, NY, The Guilford Press.

Courbet, D., 2003, "L'influence publicitaire en l'absence de souvenir des messages : les effets implicites de la simple exposition", Les Cahiers Internationaux de Psychologie Sociale, 57 (Mars 2003), pp. 39-51.

Courbet, D., Borde, A., Intartaglia, J. et Denis, S., 2004, "L'influence non consciente des publicités vues furtivement et aussitôt oubliées : une méthode d'étude socio-cognitive appliquée à Internet", Questions de communication, 5, pp. 83-102.

Drèze, X. et Zufryden F., 2000, Internet advertising: the medium is the difference, Consumption, Markets and Culture, 4, 1, pp. 12-20.

Eagly, A.H. et Chaiken S., 1993, The psychology of attitudes, Harcourt Brace Jovanovich.

Fazio, R. H., 2000, "Accessible Attitudes as Tools for Object Appraisal : Their Costs and Benefits", pp. 1-36, in : Maio G.R., Olson J.M. (eds), Why we Evaluate, Functions of Attitudes, NJ, Lawrence Erlbaum Associates.

Fazio, R.H., 2001, " On the Automatic activation of associated Evaluations: An overview", Cognition and Emotion, 15, 2001, pp.115-141.

Fazio, R. H and Olson M. A, 2003, Implicit Measures in Social Cognition Research: Their Meaning and Use, Annual Review of Psychology, 54, pp. 297-327.

Fazio, R.H. et Williams, C.J., 1986, "Attitude accessibility as a moderator of the attitude-perception and attitude-behavior relations: An investigation of the 1984 election", Journal of Personality and Social Psychology, 51, pp.505-514.

Fazio, R.H., et Towles-Schwen, T., 1999, "The MODE Model of Attitude-Behavoir Processes", in S. Chaiken \& Y. Tropes (Eds), Dual-Process Theories in Social Psychology, New-York, The Guildford Press, pp .97-116.

Fazio, R.H., Powell M.C. et Williams C.J., 1989, "The role of attitude accessibility in the attitude-tobehavior process", Journal of Consumer Research, 16, December 1989, 280-288.

Fazio, R.H., Roskos-Ewoldsen, D.R. et Powell, M.C., 1994, "Attitudes, perception, and attention", in P.M. Niedenthal \& S. Kitayama (Eds). The heart's eye: Emotional influences in perception and attention, San Diego: Academic Press, pp.197-216.

Fishbein, M., Ajzen, I., 1975, Belief, attitude, intention, and behavior: An introduction to theory and research, Reading, MA, Addison-Wesley

Fourquet-Courbet, M.P. et Courbet, D., 2004, " Nouvelle méthode d'étude des cognitions en réception (ECER) et application expérimentale à la communication politique ", Revue Internationale de Psychologie Sociale, 17, 3, pp.27-75

Ghiglione, R., 1986, L'homme communiquant, Paris, Armand Colin, Collection U, Série Psychologie.

Ghiglione, R., Trognon, A., 1993, Où va la pragmatique ? - De la pragmatique à la psychologie sociale Grenoble, Presses Universitaires de Grenoble, Vies Sociales.

Ghiglione, R., Kekenbosch, C. et Landré, A., 1995, L'analyse cognitivo-discursive, Grenoble: Presses Universitaires de Grenoble, La psychologie en plus.

Greenwald, A. G et Banaji M. R., 1995, "Implicit Social Cognition: Attitudes, self-esteem and stereotypes", Psychological Review, 102, 1, pp. 4-27. 
Greenwald, A.G., 1968, "Cognitive learning, cognitive response to persuasion, and attitude change", in A.G. Greenwald, T.C. Brock, T.M. Ostrom (Eds.), Psychological foundations of attitudes, San Diego, CA, Academic Press, pp.147-170.

Kruglanski, A.W et Webster, D.M, 1996, "Motivated closing of the mind: "Seizing" and "Freezing", Psychological Review, 103, pp.263-283.

Kruglanski, A.W. et Freund, T., 1983, "The freezing and unfreezing of lay-inferences: Effects on impresional primacy, ethnic stereotyping, and numerical anchoring", Journal of Experimental Social Psychology, 19, pp.448-468.

Lee A.Y. et Labroo A.A, 2004, The effects of conceptual and perceptual fluency on brand evaluation, Journal of Marketing Research, XLI, May 2004, 151-165.

Petty, R. E et Cacioppo J. T., 1986, Communication and Persuasion: Central and Peripheral Routes to Attitude Change, New York: Springer-Verlag.

Sanbonmatsu, D.M. et Fazio, R.H., 1990, "The role of attitudes in memory-based decision making", Journal of Personality and social Psychology, 59, pp.614-622.

Schuette, R. A et Fazio R. H., 1995, "Attitude Accessibility and Motivation as Determinants of Biased Processing: A Test of the MODE Model", Personality and Social Psychology Bulletin, 21, pp. 704710.

Wilson, T. D., Lindsey S. et Shooler T. Y., 2000, "A Model of Dual Attitude", Psychological Review, 107, 1, pp. 101-126.

Woltman Elpers, J. L. C. M., Mukherjee A. et Hoyer W.D., 2004, "Humor in Television Advertising : A Moment-to-Moment Analysis", Journal of Consumer Research, 31, December 2004, 592-598. 